\title{
Artículos
}

\section{El proceso de creación de una base de datos web}

\author{
Por Esperanza Marcos y Belén Vela
}

Resumen: La necesidad creciente de colocar información en la web, haciendo que esté disponible desde cualquier lugar del mundo, ha dado lugar a la proliferación de sitios web con datos, en muchas ocasiones, duplicados y mal organizados. Este tipo de organización casual, así como las redundancias de información, dificulta el mantenimiento de los sitios web provocando incoherencias, datos difícilmente accesibles, etc. La comunidad de bases de datos sabemos bien que un modo de evitar este tipo de problemas es, precisamente, aprovechar las facilidades que ellas nos brindan. Pero el proceso de creación de una base de datos web no es exactamente igual al proceso de desarrollo de una tradicional. Por ello es necesario también adaptar los métodos de desarrollo de bases de datos clásicas (tanto el proceso, como las técnicas, notaciones y modelos) a la web. En este artículo se presenta una panorámica de las principales metodologías para implementar bases de datos web y en concreto se resume la metodología Midas/db, desarrollada por el grupo Kybele de la Universidad Rey Juan Carlos, de Madrid.

Palabras Clave: Bases de datos, Web, Metodologías, Sistemas de información web, Ingeniería de datos.
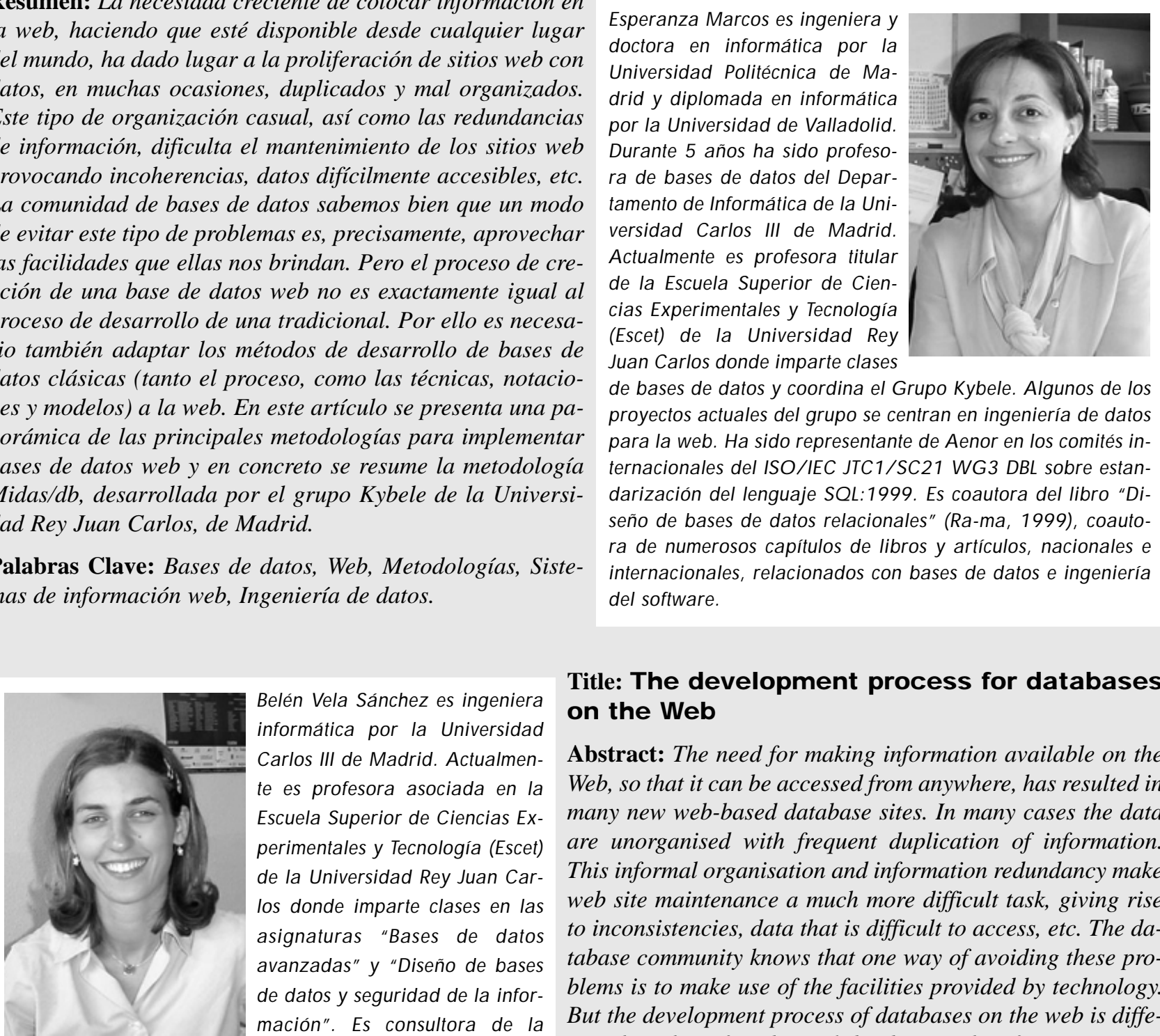

Belén Vela Sánchez es ingeniera informática por la Universidad Carlos III de Madrid. Actualmente es profesora asociada en la Escuela Superior de Ciencias Experimentales y Tecnología (Escet) de la Universidad Rey Juan Carlos donde imparte clases en las asignaturas "Bases de datos avanzadas" y "Diseño de bases de datos y seguridad de la información". Es consultora de la asignatura "Bases de datos I" en

la UOC. En la actualidad realiza su tesis doctoral en metodologías para el modelado y diseño de bases de datos web. Es coautora de diversos capítulos de libros y artículos, nacionales e internacionales, relacionados con bases de datos.

\section{Title: The development process for databases on the Web}

Abstract: The need for making information available on the Web, so that it can be accessed from anywhere, has resulted in many new web-based database sites. In many cases the data are unorganised with frequent duplication of information. This informal organisation and information redundancy make web site maintenance a much more difficult task, giving rise to inconsistencies, data that is difficult to access, etc. The database community knows that one way of avoiding these problems is to make use of the facilities provided by technology. But the development process of databases on the web is different than that of traditional databases. Therefore it is necessary to adapt the classical database development methodologies (process as well as techniques, notations and models) for working with web databases. This paper outlines the main methodologies for creating web databases and sums up the Midas/db methodology, developed by the Kybele Research Group of the University Rey Juan Carlos, in Madrid.

Keywords: Databases, Web, Methodologies, Web information systems, Data engineering.

Marcos, Esperanza; Vela, Belén. “El proceso de creación de una base de datos web”. En: El profesional de la información, 2002, julio-agosto, v. 11, n. 4, pp. 248-255.

Artículo recibido el 29-04-02

A ceptación definitiva: 03-06-02 


\section{Introducción}

El impacto que la web ha tenido en la última década ha creado la necesidad, tanto de las empresas como de otro tipo de entidades, de abrirse a este nuevo entorno. En este contexto han aparecido numerosas bases de datos (bd) accesibles a través de la web. Podemos señalar dos motivos que han propiciado que cada día existan más bd operativas en este medio. Por una parte, las empresas y organizaciones han visto la importancia de que ciertos contenidos de sus bd estuvieran disponibles a través de la Red. Además, en un primer momento, esto permitía publicar información hipermedia que podría ser recuperada desde cualquier lugar. Realizar esto era aparentemente algo tan simple como enlazar varias páginas html con el contenido deseado. Sin embargo este sistema de publicación ha dado lugar a sitios donde la información se organiza de una manera casual y habitualmente caótica, dando lugar a los ya conocidos problemas de mantenimiento, tales como: inconsistencias, información obsoleta, problemas al relacionar datos, etc.

\section{«Una vez se ha creado una bd web es posible y extremada- mente útil aprovechar la expe- riencia para tratar de sistemati- zar el proceso»}

De cualquier forma todos estos inconvenientes pueden solucionarse fácilmente si colocamos la información en una bd fácilmente mantenible y hacemos que las páginas web se generen dinámicamente extrayendo su contenido. Como sabemos, en una bd bien diseñada no habrá incoherencias y su mantenimiento es relativamente sencillo.

Una bd web es aquella cuyos datos pueden ser accesibles a través de la Red. Su mantenimiento puede realizarse o no por medio de páginas web. Crearla es parecido a hacerlo en el caso de una de carácter tradicional, pero no igual. Su concepción y desarrollo tienen algunas características específicas que han de ser tenidas en cuenta. Una vez se ha creado una bd web es posible, y extremadamente útil, aprovechar esa experiencia para tratar de sistematizar este proceso. Es por ello que en los últimos años han surgido diferentes

Agradecimientos: Las autoras quieren expresar su agradecimiento a Paloma Cáceres por algunos trabajos previos que han ayudado a la redacción de este artículo. Midas/db se ha podido llevar a cabo gracias a la financiación parcial de la Univ. Rey Juan Carlos (Pgral-2001/05) y del Mo de Ciencia y Tecnología (2FD97-2163-TIC).

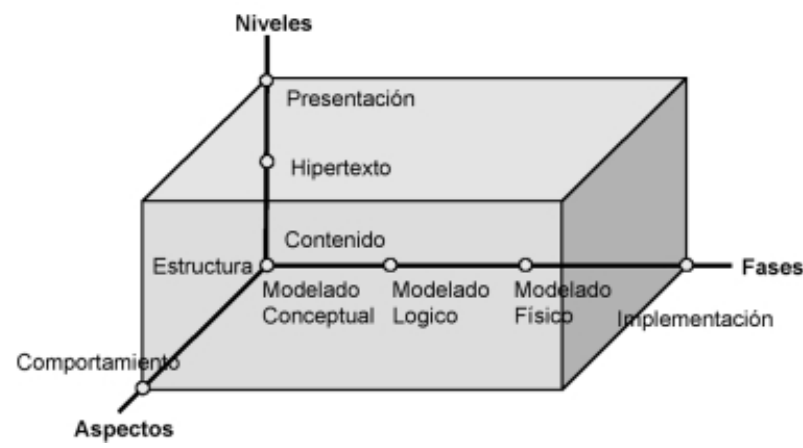

Figura 1. Dimensiones en el modelado de bd web, Schwinger y Retschitzegger (2000).

propuestas que abordan el problema de su creación desde una perspectiva de la ingeniería.

Los principales aspectos que caracterizan una metodología para su desarrollo con respecto a las correspondientes para bd tradicionales, se muestran en la clasificación tridimensional de la figura 1 (Schwinger; Retschitzegger, 2000). Como se puede apreciar, y sin tan siquiera entrar en aspectos de comportamiento, el diseño ha de realizarse a 3 niveles distintos: presentación (el diseño de las páginas), hipertexto (cómo se organiza y estructura la información de cara al usuario: enlaces, contenido de las páginas, etc.) y contenido (que corresponde a la estructuración y organización interna de la información). Para cada uno de estos niveles de organización de los datos, distinguiremos otras 3 fases previas a la implementación: modelado conceptual, lógico y físico.

En el siguiente punto llevaremos a cabo un repaso de las metodologías para la creación de bd web más significativas que podemos encontrar en la literatura; con posterioridad se resume a modo de ejemplo una concreta: Midas/db; finalizamos, en la sección 4, con las principales conclusiones.

\section{Las metodologías en el proceso de creación de una bd web}

Cuando se desarrolló en el Cern (European Organisation for Nuclear Research) a principio de los años 90 , la web fue concebida como un sistema hipermedia que permitiera a los científicos compartir información. La posibilidad de que pudiera ser consultada desde todos los lugares del mundo y por cualquier persona, fue pues su primera utilidad (Elmasri; Navathe, 2000). Esta información se publica habitualmente como páginas html que pueden contener datos en formato multimedia (audio, vídeo, etc.) y enlaces (hyperlinks) a otras permitiendo al usuario navegar de una a otra. Cuando su número es elevado el planteamiento de la estructura de navegación se convierte realmente en el diseño de una aplicación hipermedia, con la única diferencia de que ésta se ejecutará en la web. 
Por esta razón las primeras propuestas de metodologías para implementar sistemas de información web (siw) proceden del campo de la hipermedia y multimedia: HDM (Garzotto et al., 1993), RMM (Isakowitz et al., 1995), Oohdm (Schwabe; Rossi, 1998), Lowe y Hall (1999) son algunos ejemplos. HDM-lite (Fraternali; Paolini, 1998) es una adaptación de $H D M$ para el desarrollo de aplicaciones hipermedia y multimedia en la web. Debido a que uml (unified modeling language) se ha convertido en el lenguaje estándar de modelado, cabe también destacar algunos trabajos que lo extienden a fin de soportar sus propias técnicas de modelado utilizando dicho lenguaje (Baumeister et al.,1999; Koch et al., 2000; Baresi et al., 2001).

Sin embargo, estos trabajos proporcionan modelos de datos y pasos metodológicos para guiar en el proceso de diseño de aplicaciones hipermedia y multimedia, pero no tienen en cuenta el diseño y mantenimiento de los datos. Tal y como indicamos en la sección anterior, en la medida en que la cantidad de datos publicados en la web aumenta, se hace más difícil realizar un buen mantenimiento de la información contenida en las páginas html (Atzeni et al., 1999):

-En primer lugar los datos pueden cambiar y su modificación en las páginas web puede ser tediosa, ya que se encuentran interconectados con la organización del hipertexto y con la presentación gráfica del mismo.

-Por otra parte, lo más habitual será que los datos se encuentren repetidos en diferentes páginas, a fin de facilitar la legibilidad y la navegación. Por ejemplo, supongamos la información de los profesores y asignaturas de una universidad. En la página de un determinado educador figurarán las materias que imparte; además, en cada una deberán aparecer los profesores encargados de la misma. Si uno de ellos cambia de asignatura esta modificación deberá hacerse en la página del profesor y en todas y cada una de las páginas de las asignaturas afectadas por este cambio.
Esta organización da lugar a una serie de problemas como son la dificultad en encontrar y relacionar datos, errores, inconsistencias e información obsoleta derivada de un mal mantenimiento, etc. Un modo eficaz de solucionar este tipo de dificultades consiste en la utilización de bases de datos y permitir que las páginas web rellenen su contenido de forma dinámica, lo que hace posible eliminar la situación de redundancias e incoherencias anteriormente mencionada. Por este motivo, y a tener que colocar en la www información de bd ya existentes, surge la necesidad de crear aplicaciones de bd en la web y con ello nuevas propuestas metodológicas orientadas fundamentalmente a su desarrollo y mantenimiento: Araneus (Atzeni et al., 1998; Mecca et al., 1999), Autoweb (Fraternali; Paolini, 1998), Midas/db (Marcos et al., 2002).

\section{«En la medida en que la canti- dad de datos publicados en la web aumenta se hace más difí- cil realizar un buen manteni- miento de la información conte- nida en las páginas html»}

Además, cabe citar algunos métodos que aunque no son exclusivos para llevar a cabo bd web, pueden ser también útiles para este tipo de herramientas. Podrían clasificarse en dos grandes grupos:

-Marcos metodológicos que permiten abordar cualquier tipo de desarrollo (y entre ellos el de bd web) mediante la adaptación del proceso propuesto. Este es el caso de Wisdom (Castano, 2000), Midas (Cáceres; Marcos, 2001a; Cáceres; Marcos, 2001b) o webML (Bonifaci, 2000), que considera aplicaciones web con acceso móvil.

-Extensiones de metodologías clásicas, entre las que podríamos destacar las de Conallen a $u m l$ y el Proceso Unificado (Conallen, 1999; Conallen, 2000), y otras como OO-Hmethod (Gómez et al., 2000; Ca-

\begin{abstract}
Métodos Tradicionales de Modelado
\end{abstract}

$1^{\text {a }}$ Generación Métodos de Modelado de DataWeb

$2^{\mathrm{a}}$ Generación Métodos de Modelado de DataWeb

$3^{\text {a }}$ Generación Métodos de Modelado de DataWeb

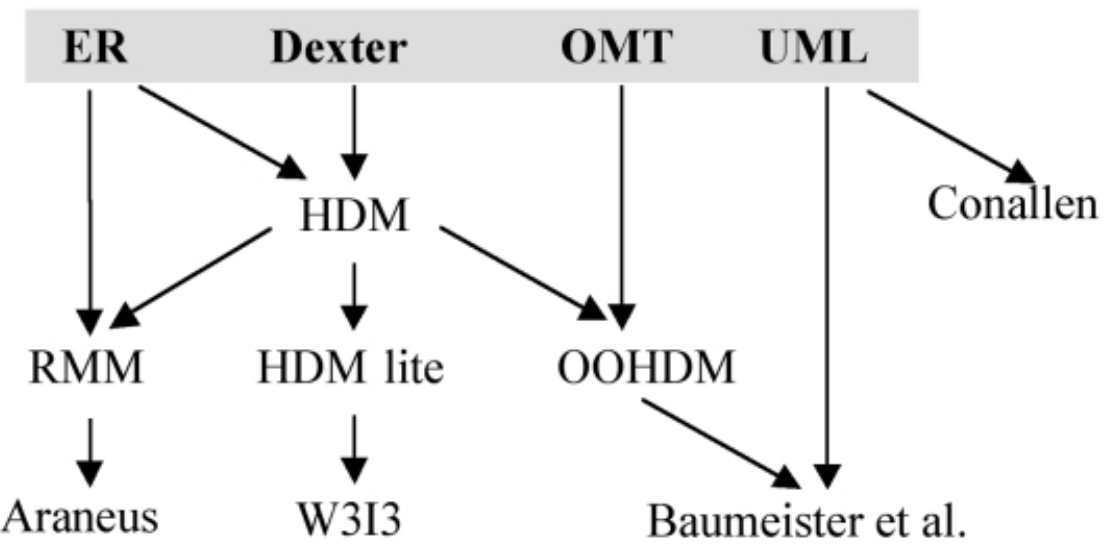

Figura 2. O rígenes de las metodologías para el modelado de bd web, Schwinger y Retschitzegger (2000). 
chero et al., 2001), elaborada en la Universidad Politécnica de Valencia. Todas ellas llevan normalmente asociadas herramientas que faciliten el desarrollo. En Fraternali (2000) puede encontrarse un estudio detallado de los principales softwares para la creación de aplicaciones web.

En Schwinger y Retschitzegger (2000) se presenta un estudio comparativo de los principales métodos de modelado de siw existentes en la actualidad. En este trabajo, tal y como se aprecia en la figura 2, se distinguen 3 generaciones en las que aparecen enmarcadas varias de las metodologías que hemos citado previamente:

- procedimientos para realizar aplicaciones hipermedia (HDM, Garzotto et al. 1993);

- se adoptan métodos de hipermedia en 3 sentidos: incorporando características del desarrollo web (HDM-lite, Fraternali; Paolini, 1998); integrando aspectos de metodologías clásicas de desarrollo software (Oohdm, Schwabe; Rossi, 1998); y teniendo en cuenta aspectos de metodologías clásicas de implementación de bd (RMM, Isakowitz et al., 1995);

- se contemplan ya cuestiones de modelado del hipertexto y de la bd: Araneus (Atzeni et al., 1998; Mecca et al., 1999), W3I3, proyecto en el que se llevó a cabo webML (Bonifaci, 2000). En esta tercera generación aparecen además extensiones de uml para el modelado de siw (Baumeister et al., 1999).

\section{Midas/db: una metodología genérica para la creación de bd web}

Puede ser aplicada a cualquier tipo de siw, y más concretamente a bd web. Propone una clasificación de siw (Cáceres; Marcos, 2000a) así como un marco metodológico que es adaptable por el diseñador según el tipo de aplicación a realizar. En este artículo nos centraremos en la parte específica para el desarrollo de bd web: Midas/db (Marcos et al., 2002).

\section{El modelo de proceso.}

Midas combina las características de los procesos para aplicaciones de hipermedia y web (iteración, incrementos y prototipado) con las nuevas propuestas de ciclos de vida ágiles, procedentes principalmente del ámbito de programación extrema (xp, extreme programming) (Beck, 1999). Las necesidades más inmediatas que tiene el cliente de aplicaciones web es que su producto software esté disponible lo antes posible. El modelo de proceso iterativo e incremental aportará las ventajas de tener productos tangibles para el cliente en versiones sucesivas, además de permitir incorporar o modificar necesidades no detectadas o no planteadas en las primeras etapas de definición del producto.

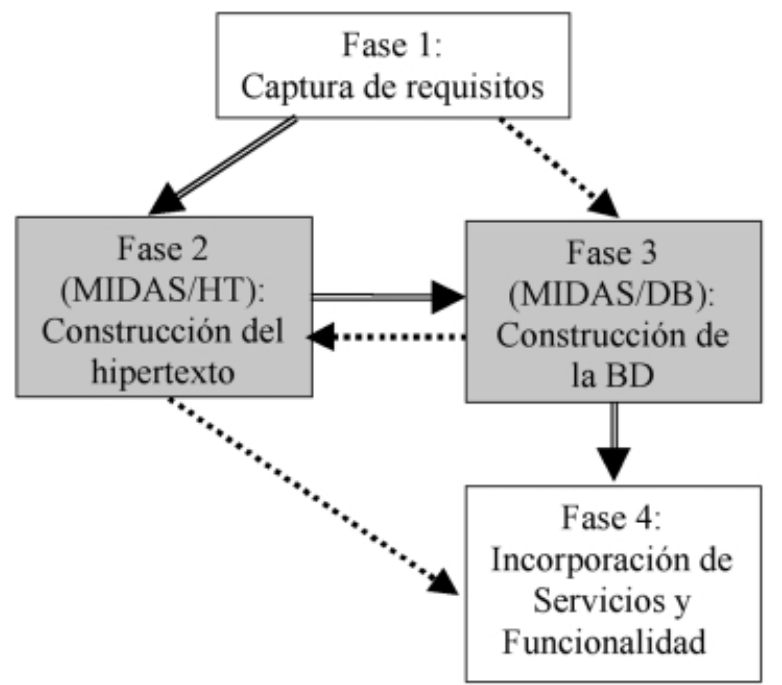

Figura 3. Proceso simplificado de la creación de un siw según Midas

Diferentes autores (Beck, 1999; Overmyer, 2000) afinan aún más, indicando que el objetivo es realizar pequeños ciclos de vida en cortos espacios de tiempo. Esto garantiza sucesivas entregas al cliente hasta completar el producto final. Por otra parte, y gracias a los prototipos, el cliente tiene la posibilidad de validar el producto continuamente.

El modelo Midas plantea realizar el producto en cuatro incrementos, en base a la caracterización de siw adoptada en Cáceres y Marcos (2000a), básica pero suficiente para identificar las actividades que deben realizarse en el desarrollo de los diferentes siw. De esta forma distingue los 3 tipos básicos de siw:

-Aplicaciones de hipertexto en la web: se trata de sitios que proporcionan información semiestructurada en páginas estáticas.

-Aplicaciones de bd web (sitios web data intensive): contienen información estructurada y semiestructurada en páginas cuyo contenido se genera dinámicamente extrayendo la información de una bd.

-Aplicaciones de funcionalidad compleja: sitios que ofrecen servicios más o menos complejos al usuario. Incluimos aquí herramientas de comercio electrónico, de educación a distancia, etc.

Midas propone realizar la aplicación en 4 incrementos: un primero para la elaboración de los requisitos y otros 3 más que coinciden con los tipos de aplicación determinados en la clasificación previa. Otros criterios tales como seguridad, arquitectura, etc., pueden considerarse ortogonales a los anteriores ya que realmente son aplicables a cualquiera de los 3 tipos de aplicación descritos. Así por ejemplo, el nivel de seguridad requerido en una aplicación web no depende de que ésta tenga o no una bd detrás, ya que podrían solicitarse los mismos procedimientos de seguridad para un sitio web en el que sólo se publicara informa- 
ción estática; por ello, estos criterios no afectan al tipo de proceso de producción software, sino que deberán ser tenidos en cuenta a lo largo del mismo, reflejándose en aquellas etapas del proceso que lo necesiten.

De este modo en una primera iteración se definirían los requisitos; en la segunda se construiría el hipertexto mediante páginas estáticas - lo que constituye la interfaz de usuario (iu) de la bd-; la tercera finalizaría con la bd y el hipertexto que ya estaría constituido por páginas dinámicas; por último, y si se tratara de una aplicación con alguna funcionalidad adicional a la propia de una bd (mantenimiento de la misma), se implementarían los servicios asociados a dicha característica.

\section{«El cliente de aplicaciones web necesita que su producto soft- ware esté disponible lo antes posible»}

La figura 3 esquematiza el proceso de creación de un siw según Midas. Aparecen sombreadas las fases específicas del proceso de creación de la bd: Midas/ht y Midas $/ d b$ (2 y 3 respectivamente del procedimiento completo de Midas). La etapa 1 equivale a la de captura de requisitos, en la que se define el sistema a desarrollar y la 3 correspondería a la implementación de servicios que permitan ofrecer alguna funcionalidad diferente de la propia de la bd. Cada una de ellas sigue un modelo de proceso iterativo con las correspondientes etapas: captura de requisitos, análisis, diseño, implementación y pruebas que, por simplicidad, no se representan en la figura.

Tal y como se puede apreciar en la imagen, la construcción del hipertexto y de la bd podría hacerse de modo paralelo, o incluso empezar por una u otra se-

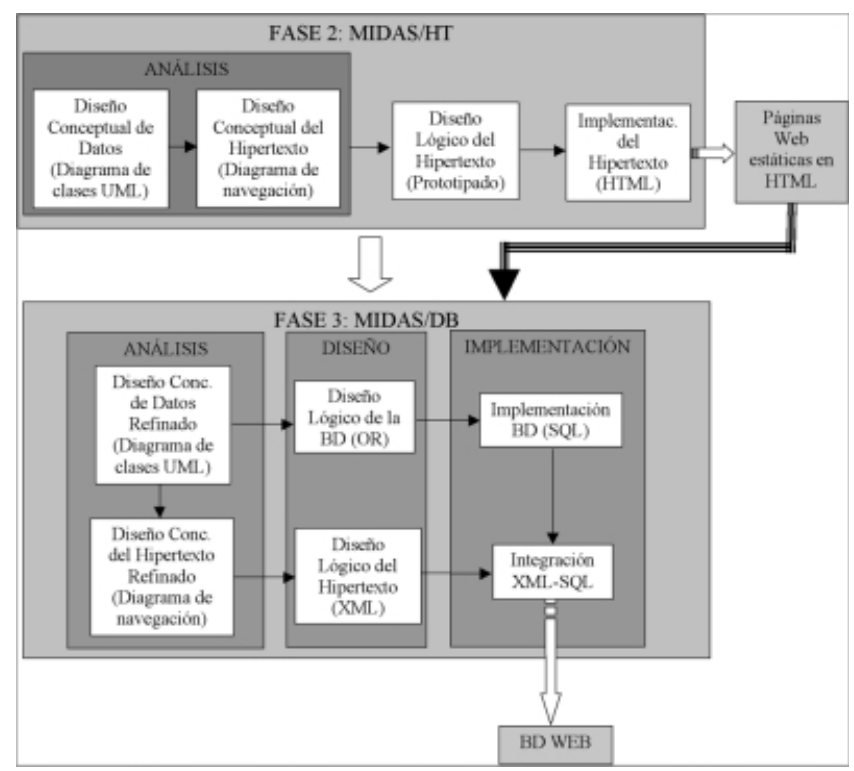

Figura 4. Fases para la creación de una bd según Midas/db gún las prioridades del sistema. Sin embargo Midas propone utilizar la siguiente secuencia (señalada en trazo continuo en la figura 3; de forma discontinua se muestran otras alternativas de proceso): fase 2: creación del hipertexto; fase 3: desarrollo de la bd; integración del hipertexto con la bd. La elección de esta sucesión se debe a los siguientes motivos:

- Por una parte se entiende que se va a crear una bd web. Es decir, consideramos que quizá el cliente no desea una cuyo contenido acabará en la web sino, más bien, que se desea construir una aplicación completa que permita publicar ciertos datos en la Red, los cuales se almacenarán en una bd. Con estas consideraciones, y siguiendo uno de los principios de los procesos ágiles, damos prioridad a aquellos aspectos que en principio consideramos fundamentales para el cliente: estar visible en la web lo antes posible; aun a pesar de que no tengamos toda la información o que la estructuración de la misma no sea la definitiva.

-Por otra parte, y dado que nuestro proceso también se basa en el prototipado rápido, esta primera versión del hipertexto (lo que realmente será la iu) no sólo permite tener una primera versión del producto en un tiempo prudencial, sino que además servirá como un prototipo que deberá ser refinado teniendo en cuenta los criterios del usuario en la siguiente fase. En este primer ciclo las páginas serán estáticas y se implementarán en html (aunque como veremos posteriormente, Midas/db propone utilizar xml para la aplicación definitiva).

-Por último, el hipertexto nos indicará también algunas pautas a la hora de diseñar nuestra bd. Como veremos a continuación Midas/db sugiere utilizar tecnología xml y objeto-relacional, la cual nos proporciona nuevos constructores permitiéndonos distintas opciones de diseño con respecto a las bd relacionales. Así, por ejemplo, una relación podría implementarse mediante las tradicionales claves ajenas o gracias a referencias (Marcos et al., 2001). Pues bien, el diseño de la navegación del hipertexto, así como otro tipo de consultas incluidas en los formularios web, pueden servirnos de guía a la hora de decidir cuándo es mejor una opción u otra.

Nos centramos en este artículo en las fases 2 y 3 , características del desarrollo de una bd web. Tal y como hemos indicado anteriormente, cada una de ellas consta de 5 actividades: captura de requisitos, análisis, diseño, implementación y pruebas. Debido a que las tareas de captura de requisitos y pruebas son igualmente aplicables a otros tipos de desarrollos web, las omitimos en el presente artículo, centrándonos en el resto. La figura 4, muestra una ampliación de las fases 2 y 3 (que aparecían sombreadas en la figura 3 ). 


\section{Fase 2: Midas/ht.}

Son dos principalmente los objetivos a alcanzar: obtener una primera versión de la aplicación que pueda estar disponible lo antes posible en la web, y servir de prototipo que permita validar con el cliente las especificaciones obtenidas en la fase 1 de captura de requisitos. Antes de la etapa de análisis existe dicha obtención de necesidades (no representada en la figura), en la que se analizarán más profundamente las exigencias de usuario específicas del hipertexto y que no habrán sido estudiadas con tanto detalle en la primera etapa. Nos referimos a requisitos de diseño de las páginas, datos concretos que se desean visualizar, formato de los mismos, navegación, etc.

Durante el análisis se realiza, en primer lugar, el diseño conceptual de datos para lo que se propone utilizar el diagrama de clases de uml (Jacobson et al., 1999). Debido a que $u m l$ se ha convertido en un lenguaje estándar de modelado proponemos su utilización, o alguna extensión del mismo siempre que sea posible. A partir de este diseño conceptual de datos se realiza el del hipertexto que incluye tanto el contenido (datos) que se visualizará en cada pantalla, como el modo de navegación entre las mismas. Para llevarlo a cabo se propone utilizar dos técnicas de RMM (Isakowitz et al., 1995; Isakowitz et al., 1998):

a. El diagrama de particiones (slice diagram), que permite representar qué datos aparecerán en qué pantallas.

b. El diagrama de navegación, que representa de dónde partirán los enlaces y hacia qué páginas irán, dónde se incluirán índices, pantallas menú, etc.

Estas técnicas se pueden representar bien en la notación propuesta por $R M M$ o, por otro lado, utilizar alguna extensión de $u m l$ como puede ser el diagrama de navegación de Baumeister et al., (1999).

Una vez realizado el diseño conceptual se pasa a las etapas de diseño lógico e implementación del hipertexto, que se realizarán conjuntamente. Para ello proponemos la técnica de prototipado rápido, utilizando alguna herramienta que permita diseñar páginas html gráficamente (Dream Weaver, Front Page, etc.). De este modo, a la vez que se realiza el diseño de la iu, se genera la primera versión de las páginas web, estáticas y en html. Una vez realizadas las pruebas correspondientes (etapa que no se visualiza en la figura 4) podrán estar disponibles en la web en un tiempo prudencial (ya que son estáticas y en html), sirviendo además de prototipo que permita validar con el usuario los requisitos iniciales de la aplicación web. En función de esta validación podrán modificarse, en la siguiente eta- pa, tanto el modelo conceptual de datos, como el del hipertexto.

\section{Fase 3: Midas/db.}

El objetivo que se pretende conseguir es obtener una bd operativa en la web. No se consideran por tanto, aspectos de funcionalidad o servicios, excepto aquellos que tienen que ver directamente con su mantenimiento y consulta.

\section{«Midas/db sugiere utilizar tec- nología xml y objeto-relacional»}

Se comienza con una etapa de captura de requisitos en la que, apoyándonos en el prototipo obtenido en la fase 2 , se revisan los requisitos iniciales con el usuario, haciendo especial hincapié en los relativos a la bd. Con esta nueva información en la etapa de análisis se refinan los modelos conceptuales, tanto el de datos como el del hipertexto. En la etapa de diseño lógico se realizan dos tareas:

a. Diseño lógico de la bd: aunque se efectúa según la tecnología escogida, en Midas/db proponemos utilizar bd (objeto-)relacionales. El modelo relacional es el más extendido y la posibilidad de utilizar extensiones de objetos permite la representación de tipos de datos complejos, información multimedia, documentos xml, etc. Proponemos hacer un diseño estándar (Vela et al., 2001), independiente del producto, utilizando para ello extensiones de uml (Marcos et al., 2001) que permiten representar un esquema objeto-relacional basado en el estándar SQL:1999 (Eisenberg; Melton, 1999). La propuesta de Marcos et al. (2001) permite también la representación en $u m l$ del esquema final de la bd en Oracle 8i.

b. Diseño lógico del hipertexto, para lo que proponemos utilizar tecnología xml. Existen varios motivos que nos llevan a esta elección: por una parte facilitará el mantenimiento de las aplicaciones al separar los aspectos de presentación de datos de la obtención y procesamiento de los mismos. Además, al tratarse de un estándar abierto e implementado ya en los principales fabricantes de software (Oracle, IBM, Microsoft, etc.), minimiza el coste de migración de las aplicaciones a otras plataformas. Una tercera razón sería que permite abrir la bd a múltiples canales (pc, $p d a$, etc.), con tan sólo utilizar unas u otras plantillas xslt según se requiera que la presentación final sea en html, wml (wireless markup language), etc. En Beleña (2002), se describe el desarrollo de una aplicación de bd web utilizando tecnología xml, así como las ventajas de utilización de dicho lenguaje.

La etapa de implementación consiste en la generación del esquema de la bd en lenguaje sql, y en su in- 
tegración con las páginas web mediante asp (active server pages), jsp (java server pages), etc. En la última etapa se realizarían las pruebas de funcionamiento final de la bd web.

\section{Conclusiones}

La creciente demanda de publicar información en la Red, así como la necesidad de organizar y estructurar la misma haciendo que los sitios web sean mas fácilmente mantenibles, ha hecho que cada vez existan más bd operativas en dicho entorno. Junto a este incremento de aplicaciones ha surgido una nueva generación de metodologías para el modelado e implementación de las mismas, con procedimientos procedentes principalmente del campo de la hipermedia y de las bd. En este artículo se ha hecho un repaso de las más relevantes para el desarrollo de siw, con especial hincapié en las específicas de bd web y presentando las principales características de una de ellas: Midas/db.

\section{«Proponemos hacer un diseño estándar, independiente del producto, utilizando para ello extensiones de uml que permi- ten representar un esquema objeto-relacional basado en la norma SQL:1999»}

En general, podemos decir que uno de los aspectos principales que diferencian la producción de una bd web con respecto a una clásica es el diseño de la iu que, en el caso que nos ocupa, será una aplicación hipermedia. Respecto al proceso proponemos realizar uno iterativo, incremental y con prototipado más apto para creaciones web en los que se espera que el producto esté disponible en la web lo antes posible (aunque no sea con su funcionalidad completa). En cuanto a tecnología la metodología expuesta se basa en estándares y propone la utilización de $u m l$ como lenguaje de modelado siempre que sea posible y de $x m l$ y bd (objeto-)relacionales; sus ventajas con respecto a otras posibles también han sido resumidas en este artículo.

Aunque en la actualidad existen diversos trabajos para la integración xml/(objeto-)relacional, quizá la principal laguna que puede encontrarse actualmente en las metodologías para la creación de bd web es la inexistencia de reglas que permitan automatizar la transición entre etapas.

\section{Bibliografía}

Atzeni, Paolo; Mecca, Giansalvatore; Merialdo, Paolo. "Design and maintenance of data-intensive web sites. En: Advances in database technology. Proceedings of the $6^{\text {th }}$ Conference on extended database techno- logy. Sheck, Hans-Jorg; Saltor, Félix; Ramos, Isidro; Alonso, Gustavo (eds.), 1998.

Atzeni, P.; Ceri, S.; Paraboschi, S.; Torlone, R. Database systems. Concepts, languages and architectures. McGraw Hill, 1999.

Baresi, L.; Garzotto, F.; Paolini, P. "Extending uml for modelling web applications". En: Proceedings of the $34^{\text {th }}$ Hawaii international conference on system sciences, Ieee Computer Society, 2001.

Baumeister, H.; Koch, N.; Mandel, L. "Towards a uml extension for hypermedia design". En: Proceedings of the UML'99. The unified modelling language-beyond the standard. France, Robert; Rumpe, Bernhard (eds.), 1999, pp. 614-629.

Beck, K. "Embracing change with extreme programming". En: Ieee computer, 1999, v. 32, n. 10, pp. 70-77.

Beleña, C. "Desarrollo de aplicaciones de gestión con pl/sql, xml y xslt". En: Revista del círculo de usuarios de Oracle de España (Cuore), 2002, febrero, pp. 9-18.

Bonifati, A.; Stefano, C.; Fraternali, P.; Maurino, A. "Building multidevice, content-centric applications using webml and the W3i3 tool suite". En: Conceptual modeling for e-bussiness and the web. Liddle, S. W.; Mayr, H. C.; Thalheim, B. (eds.), 2000, pp. 64-75.

Cáceres, P.; Marcos, E. (2000a). "Metodologías de desarrollo: estudio de su aplicabilidad para el diseño y construcción de sistemas web". En: Actas del $7^{\text {th }}$ International congress on computer science research (Ciicc'00), 2000.

Cáceres, P.; Marcos, E. (2000b). "El proceso de desarrollo en las aplicaciones web". En: Actas de las V Jornadas de ingeniería del software y bases de datos (Jisbd 2000), noviembre, 2000, pp. 59-64.

Cáceres, P.; Marcos, E. "Development methodological process for web information systems". En: Internet business and the industry of construction, Ibic'2001, 2001

Cáceres, P.; Marcos, E. "Procesos ágiles para el desarrollo de aplicaciones web. I Taller de ingeniería del software para la web". En: VI Jornadas de ingeniería del software y bases de datos, noviembre, 2001.

Castano, S.; Palopoli, L.; Torlone, R. "A general methodological framework for the development of web-based information systems". En: Conceptual modelling for e-business and the web. Liddle, S. W.; Mayr, H. C.; Thalheim, B. Lecture notes in computer science. Berlin: SpringerVerlag, 2000.

Conallen, J. "Modeling web applications architectures with uml". En: Communications of the ACM, 1999, October, n. 42, pp. 63-70.

Conallen, J. Building web applications with uml. Addison Wesley, 2000.

Eisenberg, Andrew; Melton, Jim. "SQL: 1999, formerly known as SQL3”. En: ACM Sigmod record, 1999, March, v. 28, n. 1, pp. 131-138.

Elmasri, R.; Navathe, S. B. Fundamentals of database systems. Addison-Wesley, 2000.

Fraternali, Piero; Paolini, Paolo. "A conceptual model and a tool environment for developping more scalable, dynamic and customizable web applications". En: Advances in database technology. Proceedings of the $6^{\text {th }}$ Conference on extended database technology, Sheck, Saltor, Ramos, Alonso (eds.), 1998.

Garzotto, Franca; Paolini, Paolo; Schwabe, Daniel. "HDM, a modelbased approach to hypertext application design". En: ACM Tois, 1993, January, v. 11, n. 1, pp. 1-26.

Gómez, J.; Cachero, C.; Pastor, O. "Extending a conceptual modeling approach to web application design". En: Proceedings of the $12^{\text {th }}$ International conference on advanced information systems (Caise'00). Lecture notes in computer science. Berlin: Springer-Verlag, 2000.

Gómez, J.; Cachero, C.; Pastor, O. "Conceptual modeling of device-independent web applications”. En: Ieee multimedia, 2001, v. 8, n. 2, pp. 26-39.

Isakowitz, Tomas; Stohr, Edward; Balasubramanian, Papyrus. "RMM: a methodology for structured hypermedia design". En: Соттиnications ACM, 1995, August, v. 58, n. 8, pp. 34-43. 
Isakowitz, T.; Kamis, A.; Koufaris, M. "The extended RMM methodology for web publishing". Working paper IS-98-18, Center for Research on Information Systems, 1998. Consultado en: 23-04-02. http://rmm-java.stern.nyu.edu/rmm/

Jacobson, Booch [et al.]. The unified software development process. Adison Wesley, 1999.

Koch, N.; Baumeister, H.; Mandel, L. "Extending uml to model navigation and presentation in web applications". En: Modeling web applications, workshop of the UML'2000. Winters, Geri; Winters, Jason (eds.). York, England, 2000, October.

Lowe, David; Hall, Wendy. Hypermedia \& the web. An engineering approach. J. Wiley and Sons, 1999.

Marcos, E.; Vela, B.; Cavero, J. M. "Aggregation and composition in object-relational database design". En: Advanced databases and information systems. Communications of the $5^{\text {th }}$ East-European conference, Adbis'2001.

Marcos, E.; Vela, B.; Cavero, J. M. "Extending uml for object-relational database design. En: Fourth international conference on the unified modeling language, UML'2001. Lecture notes in computer science (Lncs 2185), Springer Verlag, pp. 225-239.

Marcos, E.; Cáceres, P.; Vela, B.; Cavero, J. M. "Midas/bd: a methodological framework for web database design". En: International workshop on data semantics in web information systems. Daswis-2001, en conjunción con el $20^{\text {th }}$ International conference on conceptual modeling ER
2001. Lecture notes in computer science (Lncs), Springer Verlag, en prensa, 2002.

Mecca, G.; Merialdo, P.; Atzeni, P.; Crescenzi, V. The Araneus guide to web-site development, 1999, March. Consultado en: 04-04-02. http://poincare.inf.uniroma3.it

Overmyer, S. P. "What's different about requirements engineering for web sites?". En: Requirements engineering, 2000, n. 5, pp. 62-65.

Schwabe, D.; Rossi, G. "An object oriented approach to web-based applications design". En: Theory and practice of object system, 1998, v. 4, n. 4 , pp. $207-225$

Schwinger, W.; Retschitzegger, W. "Towards modeling of data web applications - a requirements' perspective". En: Proceedings of the America's conference on information systems, 2000, v. I, pp. 149-155.

Vela, B.; Cavero, J. M.; Marcos, E. "Diseño de bases de datos objeto-relacionales con uml". En: Anales de las Jornadas iberoamericanas de ingeniería del software e ingeniería del conocimiento, Jiisic 2001. Buenos Aires: Editorial Universidad Nacional de Jujuy, pp. 59-68.

Esperanza Marcos, Belén Vela, Grupo Kybele, Universidad Rey Juan Carlos.

Tulipán s/n, 28933, Móstoles (Madrid).

cuca@escet.urjc.es

b.vela@escet.urjc.es

\section{Complete su colección de IWE/EPI}

Deseo recibir las siguientes publicaciones:

$\square$ _-- ejemplares de la Bibliografía IWE/EPI 1992-2001 por correo postal impresos en papel (gratis).

la Bibliografía IWE/EPI 1992-2001 por correo electrónico en formato PDF (gratis).

_ álbumes de los 2 cd-roms con el texto íntegro de los primeros 100 números de la revista a 30 euros cada álbum (1 álbum es gratis para los suscriptores).

_-_ ejemplares de los siguientes números atrasados de la revista (por favor, envíenme presupuesto).

Nombre:

Institución

D pto.:

N IF institucional:

Dirección:

Código postal:

Ciudad:

País:

Teléfono: Fax:

Correo-e: @

Envíe este boletín por correo postal, fax o correo electrónico, a esta dirección:

\section{Swets Blackwell}

Caspe, 46

08010 Barcelona

España

Tel.: +34-932 701 144; fax: 932701145

mnzang@es.swetsblackwell.com 